\title{
Variable Performance and Improvement by Crossing in Commercial Populations of the Pirate Bug Orius majusculus
}

Louise B. Rasmussen ${ }^{a}$, Kim Jensen ${ }^{a, b}$, Jesper G. Sørensenc, Søren Toftc, Johannes Overgaard ${ }^{d}$, Martin Holmstrup ${ }^{b}$, Torsten N. Kristensen ${ }^{a, c}$

aDepartment of Chemistry and Bioscience, Section for Biology and Environmental Science, Aalborg University, Denmark bDepartment of Bioscience, Section for Terrestrial Ecology, Aarhus University, Denmark

garhus University, Denmark

\section{Introduction}

Organisms used in biological control are mass produced with the goal of maximizing the number of individuals (Mackauer, 1972). However, if population characteristics change over generations during rearing, this might affect the ability of biocontrol agents to control pests. There is high potential that laboratory maintained populations change characteristics over time, as several population genetic and evolutionary processes are affected during artificial rearing The fitness of a population and the efficiency of a given predator in biological control is affected by the genetic composition of the population, and founder effects might lead to inbreeding and inbreeding depression in the population (Hedrick and Kalinowski, 2000). Therefore, different populations of reared biocontrol agents may differ in quality. Furthermore, if populations are genetically depauperate, crossing populations may be advantageous as more favourable genetic combinations may appear and individuals as a consequence perform better.

\section{Conclusions}

We found that performance differences between the original populations were highly trait- and gender-specific.

Whether the crosses perform better than the original populations also appeared to depend on both the trait of investigation and on gender.

Crossbreeding might be an advantage for increasing specific performance traits in some populations.

On overall, we did not find solid evidence that crossbreeding commercial populations of $O$.

majusculus increases population performance.
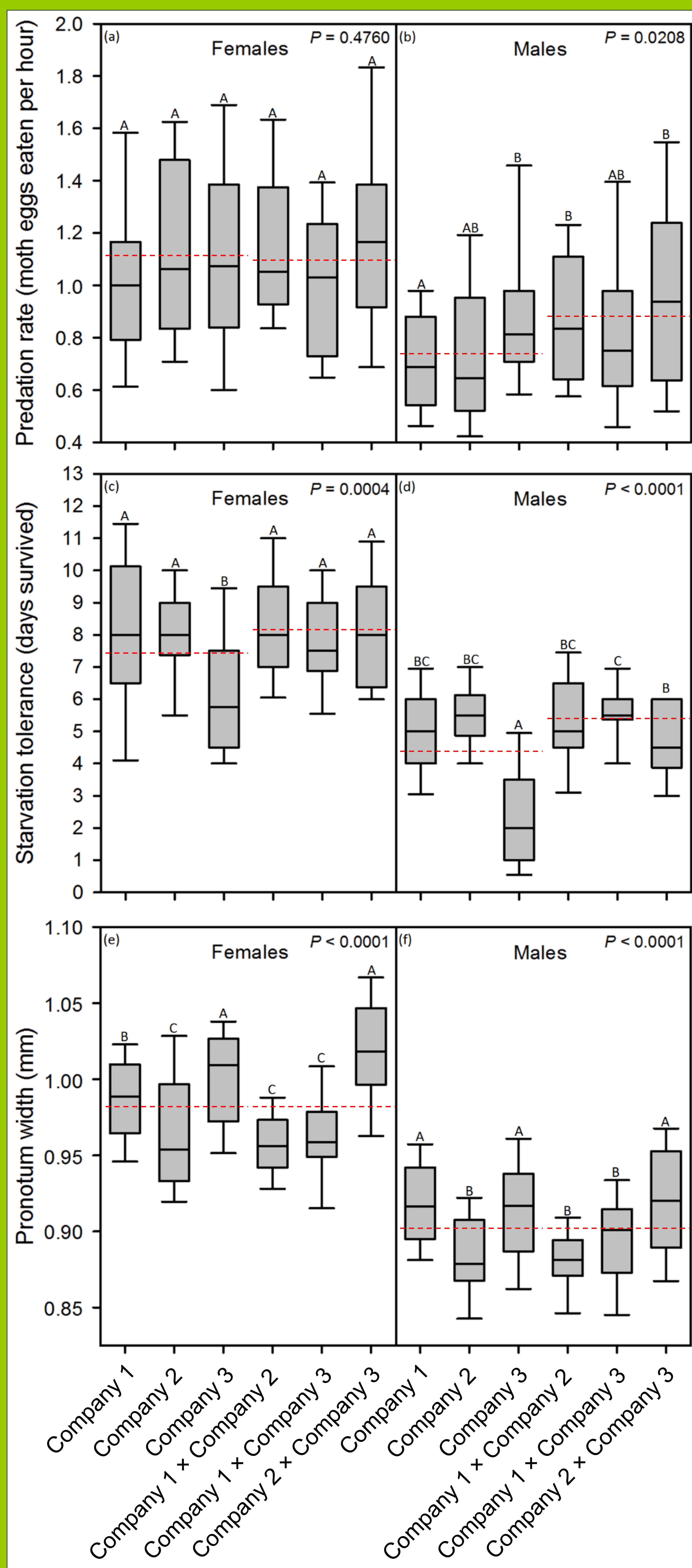

\section{Discussion}

The three original populations appeared phenotypically different with regard to some traits, whereas other traits did not significantly differ between populations. Gender differences were highly pronounced within all traits, and phenotypic differences between the populations were highly gender dependent. Females generally performed better than males across traits.

Increased fitness was indicated in some crossed populations compared to the original populations, but these differences appeared only sporadically across different traits. Contrary to our hypothesis, crossed populations therefore generally did not have higher performance scores than the original populations.

The study indicated no overall benefits of crossing populations on performance, while there was indication that some performance parameters of importance for the efficiency as biological control agents were improved in crosses

\section{Results}
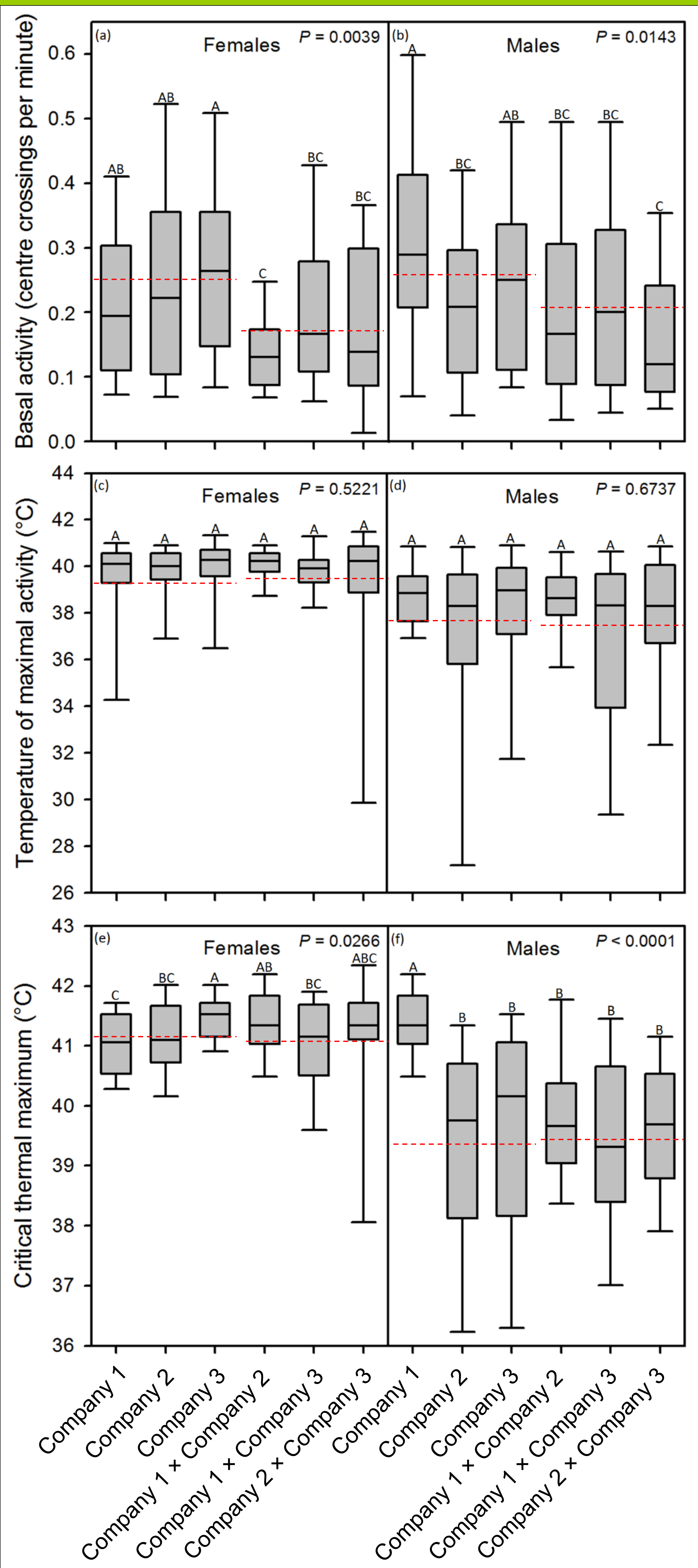